\title{
Connectome Networks: From Cells to Systems
}

\author{
Olaf Sporns
}

\begin{abstract}
Nervous systems are networks of neurons and brain regions that are structurally interconnected and dynamically linked in complex patterns. As mapping and recording techniques become increasingly capable of capturing neural structure and activity across widely distributed circuits and systems, there is a growing need for new analysis tools and modeling approaches to make sense of these rich "big data" sets. Modern network science offers a way forward. Both structural and functional brain data sets can be rendered in the form of complex networks and thus become amenable for network modeling and analysis, which can be carried out across scales, from the micro-scale of individual neurons to the macro-scale of whole-brain recordings. In this article, I sketch an overview of structural and functional brain network studies ranging from cells to systems. My emphasis will be on common themes in mapping network attributes across scales. In addition to highlighting important advances, I will outline some major challenges that need to be overcome to achieve a more complete understanding of connectome networks.
\end{abstract}

\section{Defining the Connectome}

Understanding the role of connectivity in brain function is a long-standing goal of both cellular and systems neuroscience (Sporns 2011; Schmahmann and Pandya 2007). Neuronal circuits have been at the center of anatomical and physiological investigation since the groundbreaking studies of Camillo Golgi and Santiago Ramón y Cajal in the late nineteenth century. Connectional anatomy was a core theme in early accounts of human brain function by Carl Wernicke, Theodor Meynert and Siegmund Exner that made reference to the layout and interconnectivity of brain regions and pathways. Anatomical studies that employed ever more sensitive histological staining and tracing tools and new insights into the

\footnotetext{
O. Sporns $(\triangle)$

Department of Psychological and Brain Sciences, Indiana University, Bloomington, IN 47405,

USA

e-mail: osporns@indiana.edu
} 
functioning of neurons and circuits led to theoretical notions of "neural networks." Such network models first gained momentum in the work of pioneers like Warren McCulloch and Frank Rosenblatt and ultimately transformed into "connectionism," which placed a strong emphasis on distributed processing and learning as key ingredients of neural computation.

A core theme in these historical developments is the foundational role of connectivity for brain function, an idea that has motivated the compilation of a complete connection map of the nervous system of Caenorhabditis elegans (White et al. 1986) as well as several landmark attempts to compile maps of interregional projections in the mammalian cerebral cortex (Zeki and Shipp 1988; Felleman and van Essen 1991; Young 1993). These early network maps triggered a string of theoretical and computational studies aimed at using connectivity data to define functional specialization (Passingham et al. 2002), spatial layout and wiring minimization (Chklovskii et al. 2002) as well as clusters and small-world attributes (Hilgetag et al. 2000). The importance of connectivity maps gave rise to the concept of the "connectome," first defined as "a comprehensive structural description of the network of elements and connections" of a given nervous system (Sporns et al. 2005). Several challenges were recognized right from the outset. First, brain networks span multiple spatial scales, from synaptic circuits among individual neurons all the way to whole-brain systems; integrating connectome maps across these multiple scales poses numerous conceptual and technological hurdles. Second, the connectome is changing across time as a result of neuroplasticity and development across the life span; mapping these changes requires comparative analysis of connectomes in relation to individual experience and across age. Third, connectome networks exhibit considerable variability across individuals; this structural variability may reflect individual differences in behavioral and cognitive performance. Finally, connectomics comprises a combination of structural mapping efforts and functional brain recordings, thus addressing the fundamental question of how observed brain dynamics emerge from the anatomical patterns of neural circuits.

This brief review article provides a selective overview of connectome studies that address a subset of these challenges. First, the article surveys structural mapping studies across multiple spatial scales, from connections among neurons to systems-level networks. Next, the article examines the relation of structural connectivity to dynamic brain function, including both spontaneous activity and stimulus-driven neuronal responses. The article closes with a brief summary of current efforts to use connectivity maps as key ingredients for computational models of brain function and a reflection on the status of connectomics as a foundational tool for understanding brain organization. 


\section{Brain Networks and Graph Theory}

Brain networks are collections of nodes (neuronal elements) and edges (their interconnections; Fig. 1). Empirically, brain networks are constructed from measurements of structural or functional relationships between pairs of neurons or brain regions. These pairwise relations are summarized in the form of a connection matrix that describes the relations between nodes and edges, i.e., the network's topology. Empirical methods for extracting brain network data from structural or functional measurements are continually evolving and represent an area of rapid neurotechnological innovation. Current approaches include the reconstruction of single-cell neuronal morphology and connectivity using electron or light microscopy (e.g., Helmstaedter et al. 2013), novel labeling and tract tracing approaches (e.g., Oh et al. 2014), large-scale optical recordings (e.g., Ahrens et al. 2013), and refinements of noninvasive imaging techniques (e.g., Van Essen et al. 2012).

An important distinction concerns the difference between structural and functional brain networks. Structural networks are derived from anatomical data sets and represent physical synaptic connections between neural elements, whereas functional networks are derived from neural recordings and represent their statistical relationships, e.g., covariance or cross-correlation. Structural networks are often sparse (most possible structural connections do not exist) and relatively stable across time. In contrast, functional networks undergo rapid changes in the course of both spontaneous and task-evoked neural activity and can be configured from a large number of time series analysis measures. Importantly, statements about "connectivity" in functional networks only refer to the similarity or coherence of neural time courses that may be dependent on but do not directly correspond to structural connections.

Once brain network data have been rendered in matrix form, they are amenable to an extremely wide range of statistical and modeling tools coming from network science, especially the mathematical framework of graph theory (Bullmore and Sporns 2009). A comprehensive overview of the application and interpretation of graph-theoretical approaches to brain networks is beyond the scope of this chapter [for reviews, see Rubinov and Sporns (2010), Stam (2010), and Lohmann et al. (2013)]. Briefly, descriptive measures of brain network connectivity fall into at least three different categories, reporting on different aspects of network organization. Broadly, these aspects refer to segregation, integration, and influence. Segregation and integration are best considered jointly, as they represent somewhat opposite trends towards greater functional specialization and greater functional coherence, respectively (Sporns 2013a). Graph measures of segregation (or specialization) capture the extent to which nodes aggregate into separate clusters or communities, which can be expressed by computing the network's clustering coefficient or by its tendency to form distinct modules. In contrast, measures of integration are generally aimed at quantifying the ease with which communication may occur along network paths, presumably an important aspect of how nodes can exchange information; key measures of integration relate to 


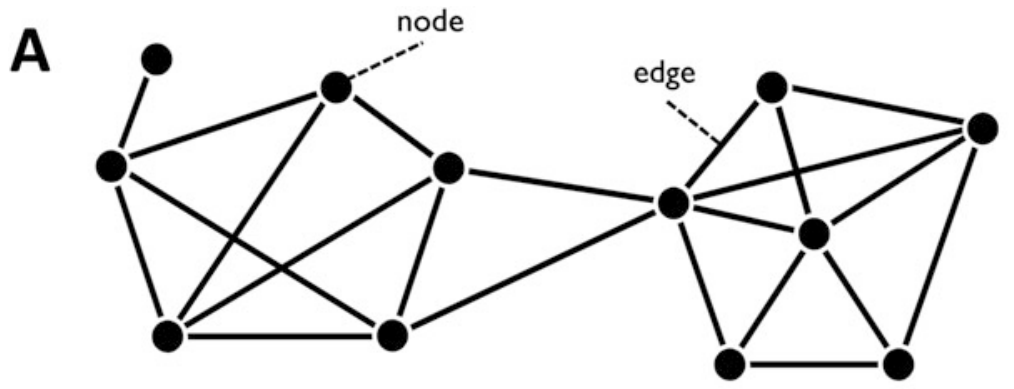

B
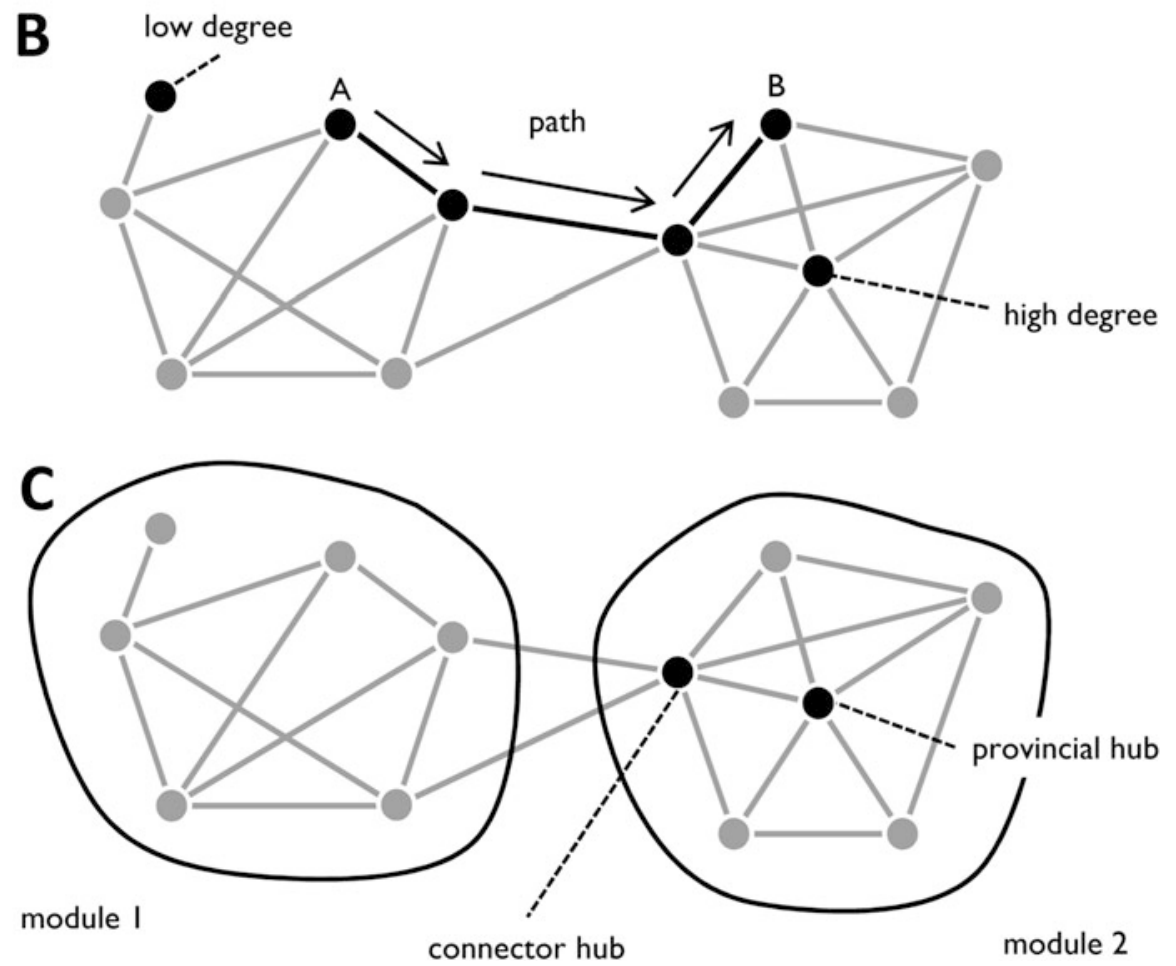

Fig. 1 Schematic illustration of a simple graph and several basic graph measures. (a) The graph represented here is binary and undirected, and it consists of a set of nodes and edges. (b) Based on the number of edges per node, some nodes can be described as low-degree and others as highdegree. Paths (sequences of edges) connect nodes to each other. In the example shown here, the shortest path linking nodes $\mathrm{A}$ and $\mathrm{B}$ consists of three edges-hence, the topological distance between A and B is three steps. (c) The network can be partitioned into two modules. Given the module partition, high-degree nodes can be classified as either connector hubs or provincial hubs. Connector hubs maintain many connections that link different modules, whereas provincial hubs mainly connect within one module only 
communication efficiency and path length. The combination of high clustering (segregation) and short path length (integration) generally indicates the presence of "small world" organization, a mode of connectivity that has been found in numerous other social, technological and biological networks (Watts and Strogatz 1998).

Measures of influence aim at quantifying the importance of network elements (nodes or edges) for the global functionality of the network, for example, by expressing their centrality in communication or their vulnerability to structural damage. Influence or centrality measures are important for detecting network "hubs." While there is no precise definition for hubs in the network literature, hubs are most often identified on the basis of their node degree (the number of distinct connections they maintain across the network) or, alternatively, through a combination of multiple nodal metrics related to connectedness and communication (Sporns et al. 2007; van den Heuvel and Sporns 2013). Hubs represent nodes of special interest in many network studies since their central embedding in the network topology makes them attractive candidates for information integration while also rendering them vulnerable to attack. In some networks (including brains), hubs can be found to be highly interconnected to form a so-called "rich club" (van den Heuvel and Sporns 2011). The concept of hubs is strongly related to modularity and network communities; hubs that predominantly link nodes within one community are also referred to as "provincial hubs," whereas hubs that interconnect multiple communities are called "connector hubs" (Fig. 1). Increasingly, cross-cutting characterizations of brain network organization that simultaneously capture segregation, integration and influence rely on decomposing networks into modules or communities that are linked by bridge connections and hub nodes. Such modular accounts of brain networks are particularly appealing since they can be applied to both structural and functional networks, and since the resulting modules have been shown to have behavioral and cognitive relevance.

Network analysis based on graph theory is prone to a number of potential limitations and pitfalls (Sporns 2014). Like all quantitative analysis, its reliability, sensitivity and reproducibility are crucially dependent on the integrity of connectivity data. This issue becomes especially important in the area of node definition, i.e., the parcellation of neural tissue into coherent areas by applying some criteria of structural or functional homogeneity. The parcellation problem (and hence node definition) continues to present pressing challenges at the meso- and macro-scales of whole-brain connectomics. In parallel, the definition of edges, particularly the estimation of structural connections using sensitive microscopic, histological or imaging techniques, continues to be problematic. Both the detection of the presence or absence of connections or pathways and the estimation of their strength or weight (based on synaptic contact area, labeling density, or tractography measures) are subject to noise, statistical biases and observational error. Significant efforts to improve neural tracing and recording techniques are currently underway, and these efforts will continue to deliver ever more accurate and more highly resolved brain network data sets. 


\section{Topology of Structural Brain Networks}

This section summarizes some recent studies reporting on the topology of structural brain networks at micro-, meso- and macro-scales. The focus is on studies that have yielded significant insights into characteristic patterns and motifs of network connectivity.

\section{Microscale}

Microscale studies of structural connectivity depend on the development of techniques for automated histology (electron microscopy or light microscopy) and reconstruction that combine sensitivity with scalability (Kleinfeld et al. 2011; Helmstaedter et al. 2011; Helmstaedter 2013). While these techniques have not yet delivered any whole-brain wiring diagrams for complex organisms, they have been successfully deployed to map specific circuits in both invertebrate and vertebrate nervous systems.

Recent studies in three model organisms (C. elegans, Drosophila, mouse) have yielded significant microscale connectivity data that have added to our knowledge of connectome architecture at the cellular level. Building on the ground-breaking work of White et al. (1986), recent studies have reported on the wiring diagram of the posterior nervous system of the $C$. elegans adult male, reconstructed from serial electron micrograph sections (Jarrell et al. 2012). Analysis of the resulting wiring pattern showed a network that was characterized by a number of features, including the presence of multiple parallel pathways that linked sensory neurons to effector neurons, some degree of recurrence within sensory systems, and the presence of structural modules. These connectional features could be related to specific aspects of sensorimotor processing and behavior. Other studies have provided additional insights for how circuit connectivity in C. elegans constrains function and behavior. Bumbarger et al. (2013) compared the synaptic connectivity of the pharyngeal nervous system of two different nematode species that exhibit very different feeding behavior. Employing graph-theoretic analyses, these behavioral differences could be traced to differences in synaptic rewiring that determined different roles of several neurons involved in regulating predatory feeding.

Studies of microscale wiring patterns in Drosophila have demonstrated that the topology of specific subcircuits can be explained on the basis of wiring length minimization and volume exclusion, both mechanisms directed at the economical conservation of space (Rivera-Alba et al. 2011). In a more recent electron microscopic study, Takemura et al. (2013) reconstructed a microscale circuit comprising 379 neurons and 8637 synapses within the optic medulla, a structure involved in visual motion detection. The circuit reconstruction revealed specific patterns of inter-neuronal connectivity that were consistent with the roles of individual neurons in generating direction selectivity. 
Detection of directed visual motion was also studied in circuits of the mouse retina, reconstructed from data obtained with serial block-face electron microscopy (Briggman et al. 2011). Analysis showed anatomically specific patterns of connectivity between amacrine and ganglion cells that were in register with physiologically measured direction selectivity of individual neurons. In subsequent work, dense reconstruction of a significant portion of the mouse retina was carried out by Helmstaedter et al. (2013). The use of a combination of manual annotation and machine learning resulted in a synaptic "contact matrix" between 950 neurons in the inner plexiform layer. Microscale connection motifs in this matrix revealed circuit mechanisms underlying motion detection and other aspects of visual function.

Direct applications of graph theory or network science methods to microscale connectivity data are still scarce, in part due to the lack of data sets comprising more than just a few neurons. Quantitative network analysis has only just begun to contribute to microscale connectome studies. Important open questions in this area concern the specificity of connections between individual neurons, the prevalence of specific network motifs that might be specialized to carry out local computations, or the presence of small world organization. As more microscale connectome data accrue, network analysis will become increasingly important for characterizing circuit models of neural computation (Denk et al. 2012).

\section{Mesoscale}

Mesoscale efforts to assemble connectivity maps for large portions or even complete nervous systems are under way in a number of organisms, with some of the most important insights coming from Drosophila and mouse.

Chiang et al. (2011) collected high-resolution 3D images of approximately 16,000 single neurons in the Drosophila brain that were then used to assemble a whole-brain connectivity matrix. Aggregation of single-neuron images into functional subdivisions, so-called "local processing units," resulted in a mesoscale connectome comprising 41 nodes and their weighted interconnections. Cluster analysis revealed distinct network communities or modules whose members were functionally specialized to carry out visual, olfactory, auditory and motor processing. Ongoing work has begun to reveal additional network attributes, including additional submodules and small-world organization (Shih et al. 2013; 2015).

Mesoscale mouse connectome projects (as well as parallel efforts in the rat cerebral cortex; Bota et al. 2015) have produced similar insights. Wang et al. (2012) performed a detailed quantitative analysis of the anatomical connections of ten areas of mouse visual cortex, including both their mutual connectivity and their external projection targets. Modularity analysis demonstrated a division of mouse cortex into two processing streams, with some anatomical and physiological data suggesting a close correspondence to the dorsal/ventral streams found in primate visual cortex. Zingg et al. (2014) generated a connectivity matrix for mouse neocortex by combining data 
from hundreds of tracer injections into a single network representation. The resulting directed connectivity network was shown to contain several modules corresponding to subdivisions or "subnetworks" involved in various sensory, motor and integrative functions. A parallel effort (Oh et al. 2014), involving high-resolution optical imaging and tracing of projections across the entire mouse brain, has resulted in another mouse connectome map that charts the directed and weighted anatomical links among 295 gray-matter regions. Initial network analysis of this map indicates the presence of high clustering as well as a number of highly connected network hubs.

Common themes in mesoscale connectomics across species are a prevalence of high clustering due to the existence of network modules, a strong association of these modules with distinct functional or behavioral domains, and the use of connection profiles of individual areas to build an understanding of their potential functional contributions. Network architectures involving modules interconnected by hubs appear to be shared among several species (invertebrate, mammalian, as well as the avian brain; see Shanahan et al. 2013).

\section{Macroscale}

The distinction between meso- and macro-scales is at present somewhat indistinct. Mesoscale maps result in connectivity data that report on areas and their inter-areal projections, as do most macroscale efforts that leverage tract tracing methods in non-human primates or noninvasive imaging in human brain. The macroscale studies summarized in this section all focus on inter-areal or large-scale projections in primate cerebral cortex.

Tract tracing has an important role to play for the study of anatomical connections in animal models, particularly in non-human primates. An extensive set of studies carried out by Henry Kennedy and colleagues (Markov et al. 2011, 2013a, b, 2014) have revealed the connectional anatomy of the macaque cerebral cortex in new detail. Injections of retrograde tracers in 29 cortical areas followed by rigorous quantification of label density across the entire cortex demonstrated a previously unknown degree of connectedness among areas. Numerous new (and mostly relatively weak) projections were uncovered, and the overall connectivity profile for each area was best approximated by a lognormal distribution (Markov et al. 2011), with a few strong projections and a large admixture of medium or weak pathways. Graph analysis provided evidence for a relatively high proportion of unidirectional links (Markov et al. 2014), a strong contribution of long-distance projections towards areal specificity (Markov et al. 2013a), significant distance-dependence of connection densities (Ercsey-Ravasz et al. 2013), and hierarchical arrangement of areas into "counter-streams" (Markov et al. 2013b). Several of these characteristic topological features are also found in other mammalian species, e.g., the cat or rodent brain. While the sensitivity and quantifiability of tract tracing data offer new opportunities for mapping connectome networks, the invasiveness of the method and the current inability to conduct whole-brain tracing across the entire network of 
pathways simultaneously impose some limitations, especially in estimating individual variability and in relating connectivity patterns to behavior.

Human brain connectomics currently relies primarily on imaging and reconstructing structural connections on the basis of diffusion MRI and tractography. This approach uses signals that record the diffusion anisotropy of water or other small molecules within biological tissue. Based on these signals, reconstruction methods then deliver inferential and statistical models of fiber anatomy. Methods for data acquisition and fiber reconstruction are under continual development, with significant recent refinements involving increased spatial resolution (Ŭgurbil et al. 2013), more robust probabilistic methods for tractography (Sotiropoulos et al. 2013) and additional measures of white matter microstructure such as axonal diameters (Alexander et al. 2010). A unique feature of noninvasive imaging methods is that they allow the acquisition of data from large numbers of individuals, thus opening opportunities for measuring individual variability and relation of connectional features to behavioral and cognitive performance, taking steps towards "population neuroscience" (Falk et al. 2013).

A large number of studies have generated network maps of the human connectome (Hagmann et al. 2008; Gong et al. 2009; Bassett et al. 2010; van den Heuvel and Sporns 2011). Network studies of human structural connectivity patterns have consistently reported broad degree distributions, with a "heavy tail" of well-connected nodes, including some that maintain very high numbers of connections. The precise shape of the degree distribution remains somewhat uncertain, due to resolution limits and issues related to node parcellation, with most studies suggesting exponential or exponentially truncated power-law distributions for node degree. An intriguing question for future work is how these distributions might compare to the log-normal profiles of connection density and weight obtained from other species (see above). Another common feature encountered across most, if not all, network studies of the human connectome is "smallworldness," i.e., the presence of high clustering and short path length (Bassett and Bullmore 2006). This is significant as the presence of small-world organization is consistent with a balance between anatomical and functional segregation on the one side (as captured by high clustering) and a simultaneous capacity for global integration on the other side (as captured by short communication paths).

High clustering in the human brain (as well as in the nervous systems of other species) is mainly driven by the presence of modules, or network communities of densely interconnected neural elements. From a network perspective, structural modules offer a connectional substrate for rapid and efficient sharing of information among restricted sets of brain regions (often found to contribute to a common set of tasks) while also promoting the functional specialization of these regions by creating boundaries that limit the spread of information across the entire network. A complementary concept is that of network hubs. As discussed earlier, hubs are regions that are less central to specific modules but instead interconnect multiple modules to each other; such hubs are generally characterized by their high degree, high centrality, and diverse connection profiles. In the human cerebral cortex, hubs have been identified in portions of the medial and superior parietal cortex as well as 
selected regions in orbitofrontal, superior frontal and lateral prefrontal cortex (Hagmann et al. 2008; Gong et al. 2009; van den Heuvel and Sporns 2013), with many of them previously described as multi- or transmodal association areas (e.g., Mesulam 1998).

Recently, several human connectivity studies have suggested a tendency for hubs to be densely interconnected in a structural core (Hagmann et al. 2008) or a "rich club" (van den Heuvel and Sporns 2011), again paralleling findings in other species (e.g., Zamora-López et al. 2010; Harriger et al. 2012). Across these different studies, a common prediction is that rich club nodes and their interconnections may have particularly important roles to play in brain communication (van den Heuvel et al. 2012). Computational studies of the human connectome have shown that a very high percentage of all short communication paths among non-rich club regions across the network must pass through the rich club. Furthermore, damage to connections that link rich club regions is predicted to have a larger disruptive effect on network communication than an equal amount of damage to connections among non-rich club regions.

Numerous common themes have emerged across different studies of primate cortex. The emerging picture is one of a modular small-world network, with clustered network communities that are interlinked by a coherent core or subnetwork (the rich club) of hub regions. The placement of the rich club within the overall network is strongly suggestive of a central role in global information flow and integration. The implications of such a structural core or rich club for cognition and behavior remain largely unexplored. One important conceptual link is that between rich-club organization and theories of "global workspace" in relation to cognition and consciousness (Dehaene and Changeux 2011). Workspace theories postulate mechanisms for integration across sensory, motor and cognitive domains that may require a dense subnetwork of distributed hub regions, i.e., the presence of a cortical rich club.

\section{Comparison Across Scales and Challenges}

How do connectome mapping efforts across different scales relate to each other? For the purpose of mapping whole nervous systems that are small and compact (such as those of many invertebrates) as well as for elucidating connectivity of local circuits in more complex brains, microscale approaches to structural connectomics are clearly of major importance. However, it seems unlikely that the application of microscale connectomics technology, even if successful across the whole brain, will ever entirely remove the need for measuring connectivity at coarser spatial scales. For descriptions of brain connectivity in large brains (e.g., in mammalian species), mesoscale and macroscale maps will remain essential as they allow establishing relations between connectivity and behavior. In addition, meso- and macroscale in vivo mapping strategies such as noninvasive neuroimaging, despite limits on resolution and various methodological biases, make an important 
contribution by drawing links between individual variations in connectivity and individual differences in cognitive and behavioral performance. It is difficult to imagine at present how microscale approaches alone can address these important research goals of connectomics in the foreseeable future.

Several challenges for structural connectomics remain. As the field matures, there will be a growing need for annotation of connectome maps with additional physiological parameters, for example, data on connectional microstructure, neurotransmitter receptors, plasticity and neuromodulatory effects, all aspects that are crucial for interpreting the functional role of connectional topology. The goal of using connectome data for explaining and predicting the operation of neuronal circuits and populations requires the inclusion of these physiological features of connections that are known to have important impacts on how neurons interact and how circuits compute (Bargmann 2012). Another challenge is to map features of connectome topology across scales, from cells to whole-brain systems. Data on nervous systems across a range of species have demonstrated a surprising degree to which global network organization is preserved; virtually all brain network data sets examined so far share some degree of high clustering, short path length, modules and hubs, and even rich-club organization. It is unknown at present if similarities exist also across different scales within the same nervous system, for example, long-range pathways between brain areas as well as local cortical circuits.

\section{Relations Between Structure and Function}

Structure-function relationships are crucial for achieving a deeper understanding of biological processes. In line with this view, the relation of structural to functional connectivity offers a key motivation for mapping connectome networks. A number of studies across micro, meso and macro scale have suggested that patterns of structural connections are indeed instrumental in shaping the dynamics of neural activity.

\section{Microscale}

The relations between circuit topology, neural computation and behavior are still relatively unexplored. Significant inroads have been made in the network anatomy of specific subregions of the C. elegans nervous system and its relation to specific behaviors (see above). Another area where detailed reconstructions of cell morphology and circuit anatomy have helped understand circuit function is motion detection (Borst and Euler 2011), specifically motion detection circuits of Drosophila and the mouse retina (see above).

Important insights have been gained from microscale studies that are built on a combined structure-function approach. An example is an analysis of anatomy and 
physiology of a subset of neurons in primary visual cortex of the mouse carried out by Bock et al. (2011). First, the authors characterized functional properties of neurons, such as their preferred stimulus orientation, using optical imaging. Then, they performed serial sectioning electron microscopy of the same tissue volume to map and reconstruct synaptic interconnections, eventually resulting in a network graph. Detailed analysis of the final connection diagram revealed some specific connectional features such as convergence of inputs from multiple pyramidal cells with diverse orientation preference onto inhibitory neurons. This pattern of convergence, while unrelated to the physiological specializations of the presynaptic cells, was partially predicted by axonal geometry, specifically the pair-wise spatial overlap of their synaptic boutons. Together with the study of Briggman et al. (2011) on direction selectivity in mouse retina (see above), this work represents an example of how the combined analysis of anatomy and physiology can inform neural accounts of computations that relate to behavior.

Large-scale recording methods applied to organisms such as the zebrafish larva can yield whole-brain recordings of highly resolved neural population activity (Ahrens et al. 2013). This dynamic circuit activity can be analyzed with time series methods, and there is evidence of functionally coherent circuits forming clusters or modules (Portugues et al. 2014). A near-term goal will be to relate the timing of correlated neural events to underlying anatomical connections that modulate whole-brain functional connectivity. Furthermore, modern molecular tools open the possibility to not only monitor but also manipulate circuit activity, for example, through the use of optogenetics (Portugues et al. 2013). This might eventually allow for uncovering causal (directed or effective) relationships between circuit elements, an aspect of connectome studies that is currently difficult to attain at the meso- and macroscales.

\section{Mesoscale}

Mesoscale studies of structural and functional connectivity have so far largely been carried out in the non-human primate. However, the increasing availability of mesoscale connectome data (e.g., Oh et al. 2014) as well as high-resolution functional MRI recordings (Mechling et al. 2014) may soon offer an opportunity to explore the issue in the mouse brain. Some important work in this area has been carried out in the macaque monkey.

Wang et al. (2013) studied the relationship between structural and functional connectivity at high spatial resolution within the monkey somatosensory cortex. Their focus of study was on connectivity within two specialized areas of the squirrel monkey somatosensory cortex (areas $3 b$ and 1), both containing representations of the monkey's body surface, specifically the tips of the digits of the monkey's hand. Resting-state functional connectivity was recorded using high-field strength functional magnetic resonance imaging (fMRI) and revealed topographically precise coupling between corresponding digits across both areas, as well as within area $3 \mathrm{~b}$. 
This pattern matched anatomical connectivity patterns observed after injections of anatomical tracers into specific digit representations. Overall, connectivity within the squirrel monkey somatosensory cortex appears to be organized anatomically and functionally in highly similar patterns, with two main "axes of information flow." One axis predominantly links representations of matched digits in area $3 \mathrm{~b}$ to area 1, whereas the other axis links representations of different digits within area $3 b$.

At the whole-brain level, a study of functional connectivity driven by spontaneous neural activity in the macaque monkey cortex by Vincent et al. (2007) found that patterns of coherent spontaneous blood oxygenation level dependent (BOLD) fluctuations were similar to patterns of anatomical connectivity derived from tract tracing studies. Adachi et al. (2012) performed a similar analysis, comparing structural and functional connectivity across 39 regions of macaque cortex and demonstrating a significant statistical relationship. More detailed analysis of functional connectivity patterns demonstrated that strong coupling among brain regions could be observed even if no direct anatomical connection was present. These indirect functional relationships were found to be due to the flow of signals along indirect structural paths and other, more complex network-wide coupling effects. Both direct and indirect couplings could be successfully captured in computational models. Taken together, these findings further support a mechanistic role of structural connections in generating organized patterns of neural dynamics.

\section{Macroscale}

At the macroscale, comparisons of structural and functional connectivity have largely centered on spontaneous or endogenously driven neural activity. In human imaging, much of the emphasis over the past several years has been on fluctuations in BOLD activity in the human brain acquired during a "task-free" or resting state. Despite its unconstrained nature, numerous studies have shown that spatial and temporal patterns of resting brain activity can be richly informative about the brain's functional organization (Raichle 2011; Buckner et al. 2013). Resting-state functional connectivity is generally expressed as the cross-correlation of time series of BOLD signals recorded with fMRI across the whole brain.

Direct comparison of resting-state functional connectivity and structural connectivity (connectome) networks has revealed robust and reproducible statistical relationships, giving rise to the idea that structural connections shape functional connectivity. A systematic analysis of structural and functional connectivity in a small cohort of human participants used a parcellation of the cortex into approximately 1000 equal-sized regions of interest (Hagmann et al. 2008). The study reported robust correlations between the strengths of structural and functional connectivity across the entire cortical surface. A more detailed analysis of the same data set (Honey et al. 2009) demonstrated that this correlation persisted even after potential confounds such as spatial proximity between regions were 
taken into account. The analysis also showed that indirect structural connections could account for a significant proportion of the functional connectivity observed between node pairs lacking direct linkage. This finding strongly suggested that functional connectivity may be partly due to the passing on of indirect influence along multi-step paths in the connectome (see Adachi et al. 2012). A parallel analysis (Skudlarski et al. 2008) also reported robust structural connectivityfunctional connectivity correlations based on a voxel-by-voxel structural connectivity-functional connectivity comparison across the cerebral cortex.

Following these early analyses, numerous independent studies have confirmed the existence of robust and significant statistical relationships between structural and (resting state) functional connectivity in the human brain (e.g., Hermundstad et al. 2013). Several studies have focused on the role of spatial embedding (i.e., the distance dependence apparent in both structural and functional connections) for shaping the topology of structural and functional connections (e.g., Vértes et al. 2012; Samu et al. 2014). Other studies have compared structure-function relations across species (Miranda-Dominguez et al. 2014). The notion that structural connections shape and/or constrain functional connections is not only supported through comparisons of anatomical and functional connectivity but is also reinforced by interventional studies that have reported changes in functional connectivity resulting from manipulations of the anatomical substrate (Johnston et al. 2008; O'Reilly et al. 2013). Extending this notion to brain and mental disorders, a large number of studies have attempted to link dysregulation of functional connectivity patterns to underlying disturbances of structural connectivity, e.g., in disrupted hub or rich club connections [reviewed in van den Heuvel and Sporns (2013)].

\section{Comparison Across Scales and Challenges}

In summary, there is converging evidence suggesting that the connectional anatomy of neurons and brain regions is shaping or constraining the statistical dependencies that emerge as neurons and brain regions become functionally activated. Many studies have relied on simple measures of dependency (such as cross-correlation or covariance) to demonstrate this relationship. A future challenge is to develop and deploy more complex and specific measures, for example, measures that are based on partial correlations or directed influence, to better distinguish statistical dependencies due to transitive (correlative) couplings from others that are mediated by direct structural connections (and hence more causal in nature). Another challenge is to observe brain dynamics at both circuit and whole-brain levels, which is currently impossible with most standard recording techniques that either suffer from a limited "field of view" (recording only very few neurons in great detail) or limited spatial and temporal resolution (e.g., noninvasive imaging). Combining whole-brain coverage with fine spatial and temporal detail would allow capturing dynamic activity unfolding within a brain's structural connectome, perhaps even in 
relation to behavior. Promising steps in this direction have been made, with the development of new activity-dependent probes and of whole-brain recordings in C. elegans and zebrafish (see above).

\section{Future Perspectives}

As the many contributions to this volume document, research in micro-, meso- and macroconnectomics is rapidly expanding and is offering a fresh perspective on brain function as emerging from the structure and dynamics of complex networks (Sporns 2011). The future of the field is difficult to predict. Extrapolating from the past, it seems likely that connectomics will be strongly influenced by new methodological and analytic developments in both data acquisition and analysis. In this final section of the article, I attempt to forecast some of the areas where the field of connectomics may make important contributions in the near future.

Computational models will play an increasingly important role, for example, in attempts to use connectome data to inform computational models of brain function and dynamics (Fig. 2). A series of such models have been used to investigate the structural basis of spontaneous or resting-brain functional connectivity as recorded with fMRI [reviewed in Deco et al. (2011)]. Model design generally combines sets of biophysical equations that specify the dynamics of neurons or neuronal populations with sets of coupling terms (for example, structural couplings specified by a connectome map). Model analysis proceeds by using some of the same time series measures (e.g., cross-correlations between neural activity patterns) that are also employed in empirical studies. Key findings coming from this modeling work include robust relations between empirical and simulated functional networks (Honey et al. 2007, 2009; Adachi et al. 2012), as well as an important role for conduction delays and noise in generating realistic resting-brain dynamics (Deco et al. 2009). This connectome-based modeling framework can be extended to include anatomically detailed models of dynamic effects induced by focal brain lesions (Alstott et al. 2009) or degeneration of brain connectivity (de Haan et al. 2012). While biophysically based models can generate simulations of rich brain dynamics, simpler models that are based on structural graph measures (Goñi et al. 2014) and/or models of diffusive processes (Abdelnour et al. 2014) and routing (Mišić et al. 2014) are gaining in importance due to their computational simplicity and analytic transparence.

Another challenge concerns the realization that brain networks are not static in time; instead, they exhibit dynamic changes on multiple time scales. Tracking such network dynamics across time presents major methodological and analytic hurdles. Networks change on slow time scales, for example, across development and the human life span, and a growing number of imaging studies are directed at characterizing the processes that guide network growth and maturation, as well as the changing distributions of hubs and network communities (e.g., Power et al. 2010). In addition to these slow changes across time, networks change on much faster time 
A structural connectivity

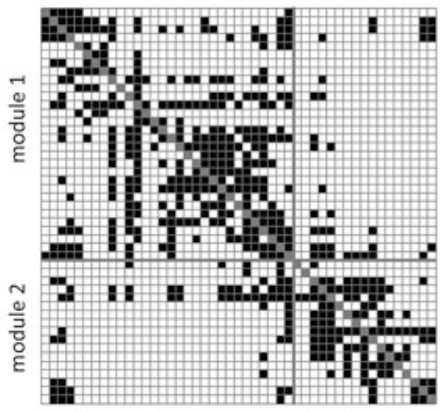

structural perturbations
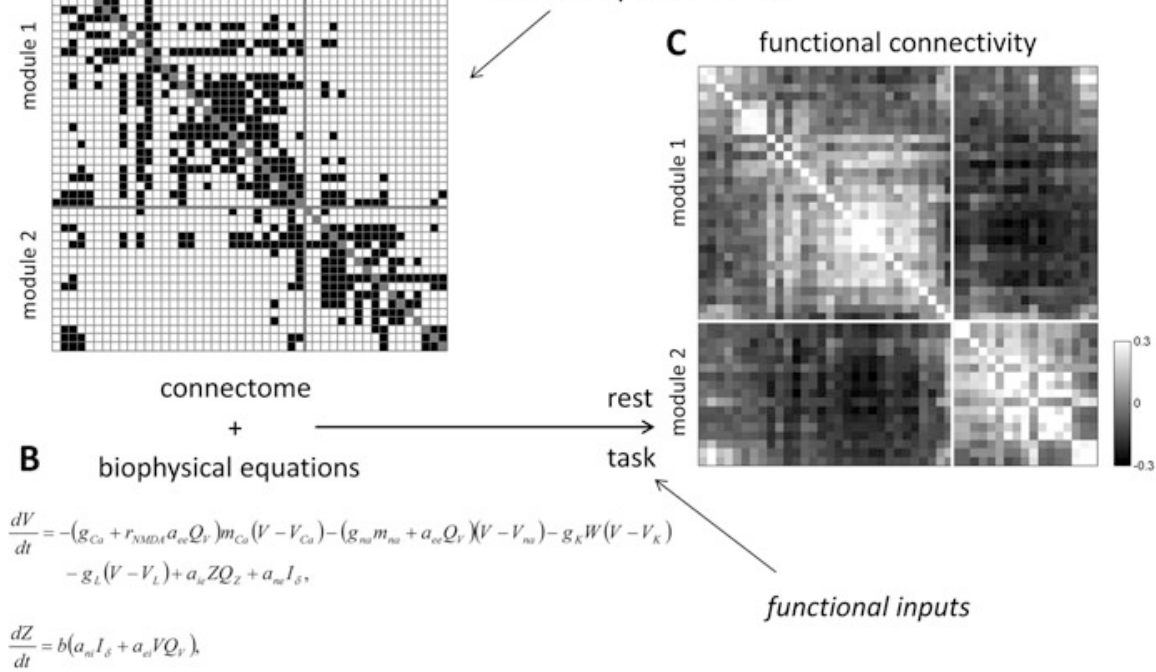

Fig. 2 An example of modeling the structure/function relationship in brain networks. (a) A structural connectivity matrix comprising 47 regions of the macaque cortex and their anatomical relationships, based on a collation of tract-tracing data [for more detail, see Honey et al. (2007)]. The matrix is binary and directed, with black squares indicating the presence of a connection from one area (matrix row) to another (matrix column). (b) Biophysical equations that describe nodal dynamics in a so-called neural mass model (Honey et al. 2007). (c) Combination of the structural connectivity matrix and the biophysical equations yields a time series for neuronal dynamics that can be rendered as functional connectivity. Structural perturbations (e.g., deletion of nodes or edges in the connectome) or functional inputs (e.g., simulating task performance) can be used to explore differences in functional connectivity. Both structural and functional connectivity are presented with identical arrangements of brain regions, and two functional modules are indicated (modules 1 and 2)

scales (seconds and milliseconds), both during "resting state" (more appropriately conceptualized as the "restless brain"; Raichle 2011) and in transitions between tasks. Informed by new approaches to network dynamics coming from network science (e.g., Mucha et al. 2010), recent studies have attempted to measure fast changes in network topology in brain recordings (Bassett et al. 2013).

In conclusion, as small data give way to "big data" in neuroscience (much of it coming from the domains of structural and functional connectivity), connectomics is likely to expand significantly in coming years. Several large-scale national and international projects and consortia directed at brain science are underway, including the Human Connectome Project and the BRAIN initiative in the U.S. as well as the Human Brain Project in the E.U. As these projects progress, there will be an increasing need for a theoretical framework that can underpin and help make sense of "big brain data" (Sporns 2013b). One promising candidate for such a framework 
is the science of networks, with its many applications in the brain across different scales and systems.

Acknowledgment The author's work was supported by the J.S. McDonnell Foundation

Open Access This chapter is distributed under the terms of the Creative Commons AttributionNoncommercial 2.5 License (http://creativecommons.org/licenses/by-nc/2.5/) which permits any noncommercial use, distribution, and reproduction in any medium, provided the original author(s) and source are credited.

The images or other third party material in this chapter are included in the work's Creative Commons license, unless indicated otherwise in the credit line; if such material is not included in the work's Creative Commons license and the respective action is not permitted by statutory regulation, users will need to obtain permission from the license holder to duplicate, adapt or reproduce the material.

\section{References}

Abdelnour F, Voss HU, Raj A (2014) Network diffusion accurately models the relationship between structural and functional brain connectivity networks. Neuroimage 90:335-347

Adachi Y, Osada T, Sporns O, Watanabe T, Matsui T, Miyamoto K, Miyashita Y (2012) Functional connectivity between anatomically unconnected areas is shaped by collective network-level effects in the macaque cortex. Cereb Cortex 22:1586-1592

Ahrens MB, Orger MB, Robson DN, Li JM, Keller PJ (2013) Whole-brain functional imaging at cellular resolution using light-sheet microscopy. Nat Methods 10:413-420

Alexander DC, Hubbard PL, Hall MG, Moore EA, Ptito M, Parker GJ, Dyrby TB (2010) Orientationally invariant indices of axon diameter and density from diffusion MRI. Neuroimage 52:1374-1389

Alstott J, Breakspear M, Hagmann P, Cammoun L, Sporns O (2009) Modeling the impact of lesions in the human brain. PLoS Comput Biol 5:e1000408

Bargmann CI (2012) Beyond the connectome: how neuromodulators shape neural circuits. Bioessays 34:458-465

Bassett DS, Bullmore ET (2006) Small world brain networks. Neuroscientist 12:512-523

Bassett DS, Brown JA, Deshpande V, Carlson JM, Grafton ST (2010) Conserved and variable architecture of human white matter connectivity. Neuroimage 54:1262-1279

Bassett DS, Porter MA, Wymbs NF, Grafton ST, Carlson JM, Mucha PJ (2013) Robust detection of dynamic community structure in networks. Chaos 23:013142

Bock DD, Lee WCA, Kerlin AM, Andermann ML, Hood G, Wetzel AW, Yurgenson S, Soucy ER, Kim HS, Reid RC (2011) Network anatomy and in vivo physiology of visual cortical neurons. Nature 471:177-182

Borst A, Euler T (2011) Seeing things in motion: models, circuits, and mechanisms. Neuron 71: 974-994

Bota M, Sporns O, Swanson LW (2015) Architecture of the cerebral cortical association connectome underlying cognition. Proc Natl Acad Sci USA 112:E2093-E2101

Briggman KL, Helmstaedter M, Denk W (2011) Wiring specificity in the direction-selectivity circuit of the retina. Nature 471:183-188

Buckner RL, Krienen FM, Yeo BT (2013) Opportunities and limitations of intrinsic functional connectivity MRI. Nat Neurosci 16:832-837

Bullmore E, Sporns O (2009) Complex brain networks: graph theoretical analysis of structural and functional systems. Nat Rev Neurosci 10:186-198

Bumbarger DJ, Riebesell M, Rödelsperger C, Sommer RJ (2013) System-wide rewiring underlies behavioral differences in predatory and bacterial-feeding nematodes. Cell 152:109-119 
Chiang AS, Lin CY, Chuang CC, Chang HM, Hsieh CH, Yeh CW, Shih CT, Wu JJ, Wang GT, Chen YC, Wu CC, Chen GY, Ching YT, Lee PC, Lin CY, Lin HH, Wu CC, Hsu HW, Huang YA, Chen JY, Chiang HJ, Lu CF, Ni RF, Yeh CY, Hwang JK (2011) Three-dimensional reconstruction of brain-wide wiring networks in Drosophila at single-cell resolution. Curr Biol 21:1-11

Chklovskii DB, Schikorski T, Stevens CF (2002) Wiring optimization in cortical circuits. Neuron 34:341-347

de Haan W, Mott K, van Straaten EC, Scheltens P, Stam CJ (2012) Activity dependent degeneration explains hub vulnerability in Alzheimer's disease. PLoS Comput Biol 8:e1002582

Deco G, Jirsa V, McIntosh AR, Sporns O, Kötter R (2009) Key role of coupling, delay, and noise in resting brain fluctuations. Proc Natl Acad Sci USA 106:10302-10307

Deco G, Jirsa VK, McIntosh AR (2011) Emerging concepts for the dynamical organization of resting-state activity in the brain. Nat Rev Neurosci 12:43-56

Dehaene S, Changeux JP (2011) Experimental and theoretical approaches to conscious processing. Neuron 70:200-227

Denk W, Briggman KL, Helmstaedter M (2012) Structural neurobiology: missing link to a mechanistic understanding of neural computation. Nat Rev Neurosci 13:351-358

Ercsey-Ravasz M, Markov NT, Lamy C, Van Essen DC, Knoblauch K, Toroczkai Z, Kennedy H (2013) A predictive network model of cerebral cortical connectivity based on a distance rule. Neuron 80:184-197

Falk EB, Hyde LW, Mitchell C, Faul J, Gonzalez R, Heitzeg MM, Keating DP, Langa KM, Martz ME, Maslowsky J, Morrison FJ, Noll DC, Patrick ME, Pfeffer FT, Reuter-Lorenz PA, Thomason ME, Davis-Kearn P, Monk CS, Schulenberg J (2013) What is a representative brain? Neuroscience meets population science. Proc Natl Acad Sci USA 110:17615-17622

Felleman DJ, van Essen DC (1991) Distributed hierarchical processing in the primate cerebral cortex. Cereb Cortex 1:1-47

Gong G, He Y, Concha L, Lebel C, Gross DW, Evans AC, Beaulieu C (2009) Mapping anatomical connectivity patterns of human cerebral cortex using in vivo diffusion tensor imaging tractography. Cereb Cortex 19:524-536

Goñi J, van den Heuvel MP, Avena-Koenigsberger A, de Mendizabal NV, Betzel RF, Griffa A, Hagmann P, Corominas-Murtra B, Thiran JP, Sporns O (2014) Resting-brain functional connectivity predicted by analytic measures of network communication. Proc Natl Acad Sci USA 111:833-838

Hagmann P, Cammoun L, Gigandet X, Meuli R, Honey CJ, Wedeen V, Sporns O (2008) Mapping the structural core of human cerebral cortex. PLoS Biol 6:e159

Harriger L, van den Heuvel MP, Sporns O (2012) Rich club organization of macaque cerebral cortex and its role in network communication. PLoS One 7:e46497

Helmstaedter M (2013) Cellular-resolution connectomics: challenges of dense neural circuit reconstruction. Nat Methods 10:501-507

Helmstaedter M, Briggman KL, Denk W (2011) High-accuracy neurite reconstruction for highthroughput neuroanatomy. Nat Neurosci 14:1081-1088

Helmstaedter M, Briggman KL, Turaga SC, Jain V, Seung HS, Denk W (2013) Connectomic reconstruction of the inner plexiform layer in the mouse retina. Nature 500:168-174

Hermundstad AM, Bassett DS, Brown KS, Aminoff EM, Clewett D, Freeman S, Frithsen A, Johnson A, Tipper CM, Miller MB, Grafton ST, Carlson JM (2013) Structural foundations of resting-state and task-based functional connectivity in the human brain. Proc Natl Acad Sci USA 110:6169-6174

Hilgetag CC, Burns GA, O’Neill MA, Scannell JW, Young MP (2000) Anatomical connectivity defines the organization of clusters of cortical areas in the macaque monkey and the cat. Philos Trans R Soc Lond B 355:91-110

Honey CJ, Kötter R, Breakspear M, Sporns O (2007) Network structure of cerebral cortex shapes functional connectivity on multiple time scales. Proc Natl Acad Sci USA 104:10240-10245

Honey CJ, Sporns O, Cammoun L, Gigandet X, Thiran JP, Meuli R, Hagmann P (2009) Predicting human resting-state functional connectivity from structural connectivity. Proc Natl Acad Sci USA 106:2035-2040 
Jarrell TA, Wang Y, Bloniarz AE, Brittin CA, Xu M, Thomson JN, Albertson DG, Hall DH, Emmons SW (2012) The connectome of a decision-making neural network. Science 337: 437-444

Johnston JM, Vaishnavi SN, Smyth MD, Zhang D, He BJ, Zempel JM, Shimony JS, Snyder AZ, Raichle ME (2008) Loss of resting interhemispheric functional connectivity after complete section of the corpus callosum. J Neurosci 28:6453-6458

Kleinfeld D, Bharioke A, Blinder P, Bock DD, Briggman KL, Chklovskii DB, Denk W, Helmstaedter M, Kaufhold JP, Lee WCA, Meyer HS, Micheva KD, Oberlaender M, Prohaska S, Reid RC, Smith SJ, Takemura S, Tsai PS, Sakmann B (2011) Large-scale automated histology in the pursuit of connectomes. J Neurosci 31:16125-16138

Lohmann G, Stelzer J, Neumann J, Ay N, Turner R (2013) "More is different" in functional magnetic resonance imaging: a review of recent data analysis techniques. Brain Connect 3: 223-239

Markov NT, Misery P, Falchier A, Lamy C, Vezoli J, Quilodran R, Gariel MA, Giroud P, ErcseyRavasz M, Pilaz LJ, Huissoud C, Barone P, Dehay C, Toroczkai Z, Van Essen DC, Kennedy H, Knoblauch K (2011) Weight consistency specifies regularities of macaque cortical networks. Cereb Cortex 21:1254-1272

Markov NT, Ercsey-Ravasz M, Lamy C, Gomes ARR, Magrou L, Misery P, Giroud P, Barone P, Dehay C, Toroczkai Z, Knoblauch K, Van Essen DC, Kennedy H (2013a) The role of longrange connections on the specificity of the macaque interareal cortical network. Proc Natl Acad Sci USA 110:5187-5192

Markov NT, Ercsey-Ravasz M, Van Essen DC, Knoblauch K, Toroczkai Z, Kennedy H (2013b) Cortical high-density counterstream architectures. Science 342:1238406

Markov NT, Ercsey-Ravasz MM, Gomes ARR, Lamy C, Magrou L, Vezoli J, Misery P, Falchier A, Quilodran R, Gariel MA, Sallet J, Gamanut R, Huissoud C, Clavagnier S, Giroud P, SappeyMarinier D, Barone P, Dehay C, Toroczkai Z, Knoblauch K, Van Essen DC, Kennedy H (2014) A weighted and directed interareal connectivity matrix for macaque cerebral cortex. Cereb Cortex 24:17-36

Mechling A, Hübner N, Lee HL, Hennig J, von Elverfeldt D, Harsan LA (2014) Fine-grained mapping of mouse brain functional connectivity with resting-state fMRI. Neuroimage 96: 203-215, http://dx.doi.org/10.1016/j.neuroimage.2014.03.078

Mesulam MM (1998) From sensation to cognition. Brain 121:1013-1052

Miranda-Dominguez O, Mills BD, Grayson D, Woodall A, Grant KA, Kroenke CD, Fair DA (2014) Bridging the gap between the human and macaque connectome: a quantitative comparison of global interspecies structure-function relationships and network topology. J Neurosci 34:5552-5563

Mišić B, Sporns O, McIntosh AR (2014) Communication efficiency and congestion of signal traffic in large-scale brain networks. PLoS Comput Biol 10:e1003427

Mucha PJ, Richardson T, Macon K, Porter MA, Onnela JP (2010) Community structure in timedependent, multiscale, and multiplex networks. Science 328:876-878

O'Reilly JX, Croxson PL, Jbabdi S, Sallet J, Noonan MP, Mars RB, Browning PGF, Wilson CRE, Mitchell AS, Miller KL, Rushworth MFS, Baxter MG (2013) Causal effect of disconnection lesions on interhemispheric functional connectivity in rhesus monkeys. Proc Natl Acad Sci USA 110:13982-13987

Oh SW, Harris JA, Ng L, Winslow B, Cain N, Mihalas S, Wang Q, Lau C, Kuan L, Henry AM, Mortrud MT, Quellette B, Nguyen TN, Sorensen SA, Slaughterbeck CR, Wakeman W, Li Y, Feng D, Ho A, Nicholas E, Hirokawa KE, Bohn P, Joines KM, Peng H, Hawrylycz MJ, Phillips JW, Hohmann JG, Wohnoutka P, Gerfen CR, Koch C, Bernard A, Dang C, Jones AR, Zeng H (2014) A mesoscale connectome of the mouse brain. Nature 508:207-214

Passingham RE, Stephan KE, Kötter R (2002) The anatomical basis of functional localization in the cortex. Nat Rev Neurosci 3:606-616

Portugues R, Severi KE, Wyart C, Ahrens MB (2013) Optogenetics in a transparent animal: circuit function in the larval zebrafish. Curr Opin Neurobiol 23:119-126 
Portugues R, Feierstein CE, Engert F, Orger MB (2014) Whole-brain activity maps reveal stereotyped, distributed networks for visuomotor behavior. Neuron 81:1328-1343

Power JD, Fair DA, Schlaggar BL, Petersen SE (2010) The development of human functional brain networks. Neuron 67:735-748

Raichle ME (2011) The restless brain. Brain Connect 1:3-12

Rivera-Alba M, Vitaladevuni SN, Mishchenko Y, Lu Z, Takemura SY, Scheffer L, Meinertzhagen IA, Chklovskii DB, de Polavieja GG (2011) Wiring economy and volume exclusion determine neuronal placement in the Drosophila brain. Curr Biol 21:2000-2005

Rubinov M, Sporns O (2010) Complex network measures of brain connectivity: uses and interpretations. Neuroimage 52:1059-1069

Samu D, Seth AK, Nowotny T (2014) Influence of wiring cost on the large-scale architecture of human cortical connectivity. PLoS Comput Biol 10:e1003557

Schmahmann JD, Pandya DN (2007) Cerebral white matter-historical evolution of facts and notions concerning the organization of the fiber pathways of the brain. J Hist Neurosci 16: 237-267

Shanahan M, Bingman VP, Shimizu T, Wild M, Güntürkün O (2013) Large-scale network organization in the avian forebrain: a connectivity matrix and theoretical analysis. Front Comput Neurosci 7:89

Shih CT, Sporns O, Chiang AS (2013) Toward the Drosophila connectome: structural analysis of the brain network. BMC Neurosci 14(Suppl 1):P63

Shih CT, Sporns O, Yuan SL, Su TS, Lin YJ, Chuang CC, Wang TY, Lo CC, Greenspan RJ, Chiang AS (2015) Connectomics-based analysis of information flow in the Drosophila brain. Current Biol 25:1249-1258

Skudlarski P, Jagannathan K, Calhoun VD et al (2008) Measuring brain connectivity: diffusion tensor imaging validates resting state temporal correlations. Neuroimage 43:554-561

Sotiropoulos SN, Jbabdi S, Xu J, Andersson JL, Moeller S, Auerbach EJ, Glasser MF, Hernandez M, Sapiro G, Jenkinson M, Feinberg DA, Yacoub E, Lenglet C, Van Essen DC, Ugurbil K, Behrens TE (2013) Advances in diffusion MRI acquisition and processing in the human connectome project. Neuroimage 80:125-143

Sporns O (2011) Networks of the brain. MIT Press, Cambridge

Sporns O (2013a) Network attributes for segregation and integration in the human brain. Curr Opin Neurobiol 23:162-171

Sporns O (2013b) Making sense of brain network data. Nat Methods 10:491-493

Sporns O (2014) Contributions and challenges for network models in cognitive neuroscience. Nat Neurosci 17:652-660

Sporns O, Tononi G, Kötter R (2005) The human connectome: a structural description of the human brain. PLoS Comput Biol 1:245-251

Sporns O, Honey CJ, Kötter R (2007) Identification and classification of hubs in brain networks. PLoS One 2:e1049

Stam CJ (2010) Characterization of anatomical and functional connectivity in the brain: a complex networks perspective. Int J Psychophysiol 77:186-194

Takemura SY, Bharioke A, Lu Z, Nern A, Vitaladevuni S, Rivlin PK, Katz WT, Olbris DJ, Plaza SM, Winston P, Zhao T, Horne JA, Fetter RD, Takemura S, Blazek K, Chang LA, Ogundeyi O, Saunders MA, Shapiro V, Sigmund C, Rubin GM, Scheffer LK, Meinertzhagen IA, Chklovskii DB (2013) A visual motion detection circuit suggested by Drosophila connectomics. Nature 500:175-181

Uğurbil K, Xu J, Auerbach EJ, Moeller S, Vu AT, Duarte-Carvajalino JM, Lenglet C, Wu X, Schmitter S, Van de Moortele PF, Strupp J, Sapiro G, De Martino F, Wang D, Harel N, Garwood M, Chen L, Feinberg DA, Smith SM, Miller KL, Sotiropoulos SN, Jbabdi S, Andersson JLR, Behrens TEJ, Glasser MF, Van Essen DC, Yacoub E (2013) Pushing spatial and temporal resolution for functional and diffusion MRI in the Human Connectome Project. Neuroimage 80:80-104 
van den Heuvel MP, Sporns O (2011) Rich-club organization of the human connectome. J Neurosci 31:15775-15786

van den Heuvel MP, Sporns O (2013) Network hubs in the human brain. Trends Cogn Sci 17: 683-696

van den Heuvel MP, Kahn RS, Goñi J, Sporns O (2012) A high-cost, high-capacity backbone for global brain communication. Proc Natl Acad Sci USA 109:11372-11377

Van Essen DC, Ugurbil K, Auerbach E, Barch D, Behrens TEJ, Bucholz R, Chang A, Chen L, Corbetta M, Curtiss SW, Della Penna S, Feinberg D, Glasser MF, Harel N, Heath AC, LarsenPrior L, Marcus D, Michalareas G, Moeller S, Oostenveld R, Petersen SE, Prior F, Schlaggar BL, Smith SM, Snyder AZ, Xu J, Yacoub E (2012) The human connectome project: a data acquisition perspective. Neuroimage 62:2222-2231

Vértes PE, Alexander-Bloch AF, Gogtay N, Giedd JN, Rapoport JL, Bullmore ET (2012) Simple models of human brain functional networks. Proc Natl Acad Sci USA 109:5868-5873

Vincent JL, Patel GH, Fox MD, Snyder AZ, Baker JT, Van Essen DC, Zempel JM, Snyder LH, Corbetta M, Raichle ME (2007) Intrinsic functional architecture in the anaesthetized monkey brain. Nature 447:83-86

Wang Q, Sporns O, Burkhalter A (2012) Network analysis of corticocortical connections reveals ventral and dorsal processing streams in mouse visual cortex. J Neurosci 32:4386-4399

Wang Z, Chen LM, Négyessy L, Friedman RM, Mishra A, Gore JC, Roe AW (2013) The relationship of anatomical and functional connectivity to resting-state connectivity in primate somatosensory cortex. Neuron 78:1116-1126

Watts DJ, Strogatz SH (1998) Collective dynamics of "small-world" networks. Nature 393: $440-442$

White JG, Southgate E, Thomson JN, Brenner S (1986) The structure of the nervous system of the nematode Caenorhabditis elegans. Philos Trans R Soc Lond B 314:1-340

Young MP (1993) The organization of neural systems in the primate cerebral cortex. Proc R Soc Lond B 252:13-18

Zamora-López G, Zhou C, Kurths J (2010) Cortical hubs form a module for multisensory integration on top of the hierarchy of cortical networks. Front Neuroinform 4:1

Zeki S, Shipp S (1988) The functional logic of cortical connections. Nature 335:311-317

Zingg B, Hintiryan H, Gou L, Song MY, Bay M, Bienkowski MS, Foster NN, Yamashita S, Bowman I, Toga AW, Dong HW (2014) Neural networks of the mouse neocortex. Cell 156:1096-1111 